\title{
SIGNS OF DISTRESS
}

At any stage of the interview if a woman:

$>$ States that she is feeling anxious, upset, experiencing distressing flashbacks, or,

$>$ Shows physical signs (e.g., crying, shaking, withdrawing, etc.) that the interview is stressful.

\section{STOP}

The interview immediately \& ASSESS.

\section{ASSESS}

Ask:

$>$ Are you okay?

$>$ Would you like to end the interview now? Take a break? Or continue with the interview?

*If the woman is no longer showing signs of distress or states that she would like to continue with the interview, follow the PROCEED pathway.

**If there are any indications that the woman is not coping or does not wish to continue with the interview follow the END pathway.

\section{*PROCEED}

If the woman is no longer demonstrating signs of distress, or states to be okay, and consents; the interview may recommence (continue to monitor for any further signs of distress for the duration of the interview).

\section{**END}

If the woman is unable to continue the interview, provide her:

$>$ With a safe/ quite space away from interview area to take time to recover.

$>$ An opportunity to discuss her concerns.

$>$ With the opportunity to call a family member or close friend (if one is not already present) for support or with consent, the principal researcher will call Refugee Health Service on behalf of the woman for phone counselling support with a community health advocate or nurse.

Once she feels safe to go home ensure that:

$>$ She has a safe way to get home,

$>$ She has a mental health support card (with useful numbers),

$>$ She has the principal researcher's contact details if she has any further concerns, and

$>$ Encourage her to contact GP or mental health provider for further support.

N.B: If the woman reveals information regarding a problematic episode of care at a healthcare facility or institution the researcher will provide the woman with the SA Health's "your feedback is important" consumer information sheet which explains how to lodge a complaint or provide service feedback.

\section{FOLLOW UP}

If the woman provides consent:

$>$ Follow up with a courtesy phone call within $48 \mathrm{hrs}$ post interview to check distress status.

$>$ Any further concerns must be directed to Lifeline (13 11 14) or Refugee Health Service (8237 3900) for support.

1. Modified from Draucker CB, Martsolf DS \& Poole C 2009, 'Developing distress protocols for research on sensitive topics', Archives of Psychiatric Nursing, vol. 23, no. 5, pp. 343-350, \& Haigh C \& Witham G 2015, 'Distress protocol for qualitative data collection', Manchester Metropolitan University, Manchester. 